\title{
Genome wide search to identify reference genes candidates for gene expression analysis in Gossypium hirsutum
}

P. K. Smitha ${ }^{1,2}$, K. Vishnupriyan ${ }^{3}$, Ananya S. Kar ${ }^{3,4}$, M. Anil Kumar ${ }^{2}$, Christopher Bathula ${ }^{3}$, K. N. Chandrashekara ${ }^{5}$, Sujan K. Dhar ${ }^{2^{*}}$ and Manjula Das ${ }^{3^{*}}$ (D)

\begin{abstract}
Background: Cotton is one of the most important commercial crops as the source of natural fiber, oil and fodder. To protect it from harmful pest populations number of newer transgenic lines have been developed. For quick expression checks in successful agriculture qPCR (quantitative polymerase chain reaction) have become extremely popular. The selection of appropriate reference genes plays a critical role in the outcome of such experiments as the method quantifies expression of the target gene in comparison with the reference. Traditionally most commonly used reference genes are the "house-keeping genes", involved in basic cellular processes. However, expression levels of such genes often vary in response to experimental conditions, forcing the researchers to validate the reference genes for every experimental platform. This study presents a data science driven unbiased genome-wide search for the selection of reference genes by assessing variation of $>50,000$ genes in a publicly available RNA-seq dataset of cotton species Gossypium hirsutum.

Result: Five genes (TMN5, TBL6, UTR5B, AT1g65240 and CYP76B6) identified by data-science driven analysis, along with two commonly used reference genes found in literature (PP2A1 and UBQ14) were taken through qPCR in a set of 33 experimental samples consisting of different tissues (leaves, square, stem and root), different stages of leaf (young and mature) and square development (small, medium and large) in both transgenic and non-transgenic plants. Expression stability of the genes was evaluated using four algorithms - geNorm, BestKeeper, NormFinder and RefFinder.
\end{abstract}

Conclusion: Based on the results we recommend the usage of TMN5 and TBL6 as the optimal candidate reference genes in QPCR experiments with normal and transgenic cotton plant tissues. AT1g65240 and PP2A1 can also be used if expression study includes squares. This study, for the first time successfully displays a data science driven genome-wide search method followed by experimental validation as a method of choice for selection of stable reference genes over the selection based on function alone.

Keywords: Reference gene, qPCR, Gossypium hirsutum, Transgenic, Data science, Cotton

\section{Background}

Real time quantitative PCR (qPCR) is the most reliable, accurate and cost-effective technique for studying differential gene expression [1]. This technique has an edge

\footnotetext{
* Correspondence: sujan@beyondantibody.com; manjula.msmf@gmail.com ${ }^{2}$ Beyond Antibody LLP, S-005 Krishna Greens, Krishna Temple Road, Dodda Bomasandra, Bangalore, Karnataka 560 097, India

${ }^{3}$ Tumor Immunology Program, DSRG1, MSCTR, MSMF, Mazumdar Shaw Medical Centre, 8th floor, Narayana Health City, Bommasandra, Bangalore, Karnataka 560 099, India

Full list of author information is available at the end of the article
}

over other commonly employed methods in gene expression like Northern blot hybridization and RT-PCR (reverse transcription polymerase chain reaction), owing to its high sensitivity, specificity, accuracy and broad dynamic range $[2,3]$. However, the major pitfalls affecting qPCR data are the variability in multiple factors such as quality and integrity of RNA samples, the efficiency of cDNA synthesis and PCR efficiency across experiments [4]. An appropriate normalization strategy like the selection of good reference genes is crucial to arrive at

(c) The Author(s). 2019 Open Access This article is distributed under the terms of the Creative Commons Attribution 4.0 International License (http://creativecommons.org/licenses/by/4.0/), which permits unrestricted use, distribution, and 
biologically meaningful gene expression results from qPCR experiments [5].

A good reference gene should have a constant level of expression across various experimental parameters $[6,7]$ or between cells of different tissues [8]. Traditionally used reference genes for qPCR studies were carryovers from semi-quantitative methods [9]. Often the expression level of many of these genes was found to be very high leading to faulty normalization for low expressing target genes in highly sensitive qPCR experiments [10]. It was cautioned to discontinue their usage for qPCR, as numerous studies began to demonstrate that the transcript levels of these genes can vary considerably under different physiological conditions [11].

The availability of genome-wide expression data through high throughput experiments like microarray has helped to identify stable genes that could be used for normalization in qPCR experiments [1]. Among the initial work done [12], the main focus was on evaluating a set of known reference gene candidates for the stability of expression using several normalization algorithms geNorm [13], NormFinder [14] and BestKeeper [15]. However, some researchers also tried assessing gene stability using the bioinformatics approach [16], statistical measures like the coefficient of variation (CV) [17] and difference in DNA entropies in promoters driving the expression of reference and tissue specific genes [18]. Assuming that true reference genes should follow Normal distribution across samples, it was attempted to discover reference genes for expression studies in Soybean using CV and $p$-value from a normality test [19]. In another attempt, an automated workflow called findRG [20] was proposed to find reference genes in different plant species and human cancers using the $\mathrm{CV}$ as the primary measure. However, the approach of using high throughput transcriptome sequencing (RNA-seq) data to identify genes with least variation has become quite popular off late as evident from a number of studies in diverse organisms as human [21], apple [22], molusc [23] and C. savignyi, a marine organism [10].

Cotton is one of the most economically important cultivated crops. It is the major source of natural fiber for the textile industry and thus an important target for genetic modification. Transgenic technology has been applied to cotton for improving the agronomic traits, tolerance to insects, resistance to herbicides and fiber qualities [24, 25]. In this study, we have used Bollgard II (Monsanto) transgenic cotton that contains two Bacillus thuringiensis genes $C r y 1 A c$ and $C r y 2 A b$ proven to have good insecticidal efficacy against Lepidopteran larvae (cotton bollworm: Helicoverpa armigera). Numerous studies have focused on identifying the most suitable reference genes for different cotton species under biotic and abiotic stresses [11, 24, 26-36]. However, the study on the effect of expression of a transgene becomes very important, as various studies have indicated an altered expression of endogenous genes because of the expression of transgenes in both plants $[37,38]$ and animals [39]. Thus, the influence of the transgene on the expression levels of the endogenous genes, especially those used as reference genes needs to be thoroughly evaluated [39] before the start of a study.

In this study, we have employed an unbiased genomewide search from a publicly available gene expression dataset for cotton to identify potential reference gene candidates to finally select the most stable genes for gene expression analysis in cotton. We have addressed expression stability of the potential candidate reference genes to be used in both non-transgenic and transgenic lines of G. hirsutum under various experimental conditions comprising of different tissues (leaves, stem and squares), age categories (1 to 3 month old plant), developmental stages of leaves (young and mature leaves) and square (small, medium and large squares). A data-driven analysis approach complemented with experimental validation used in this study can be extended to other scientific model systems with a large number of data.

\section{Results}

\section{Selection of candidate genes}

Candidate reference genes were chosen in an unbiased manner from the publicly available cotton FGD dataset (www.cottonfgd.org) containing RNA-seq FPKM values for 66,577 genes. Out of this set only 51,272 genes could be mapped to a gene name from JGI annotation available as a part of the same dataset. From this annotated set, 11,137 genes were eliminated as low-expressing genes (median FPKM $\leq 0$ ) and the analysis was carried out using the remaining 40,135 genes. Silhouette analysis indicated that only two clusters were most optimal for the analysis (Additional file 3). A representation of the two clusters in (CV, MAD, 1-p) hyperspace is shown in Fig. 1 with the details given in Table 1 .

Genes from Cluster 1 with least medoid value of each parameter was chosen over cluster 2 and sorted in ascending order based on the calculated Euclidean distance. The top 100 genes were further filtered out according to their GO annotation for subcellular location and molecular function. Gene products localizing to nucleus are more likely to be involved in transcription and can be subjected to variations under different experimental conditions leading to differential mRNA expression. Thus, non-cytoplasm expressed genes or genes involved in translation are not considered for further analysis. The entire workflow is summarized in Fig. 2 and the top ten genes considered for further analyses in Table 2. Two most popular reference genes used in cotton research under biotic stress, $U B Q 14$, a polyubiquitin 


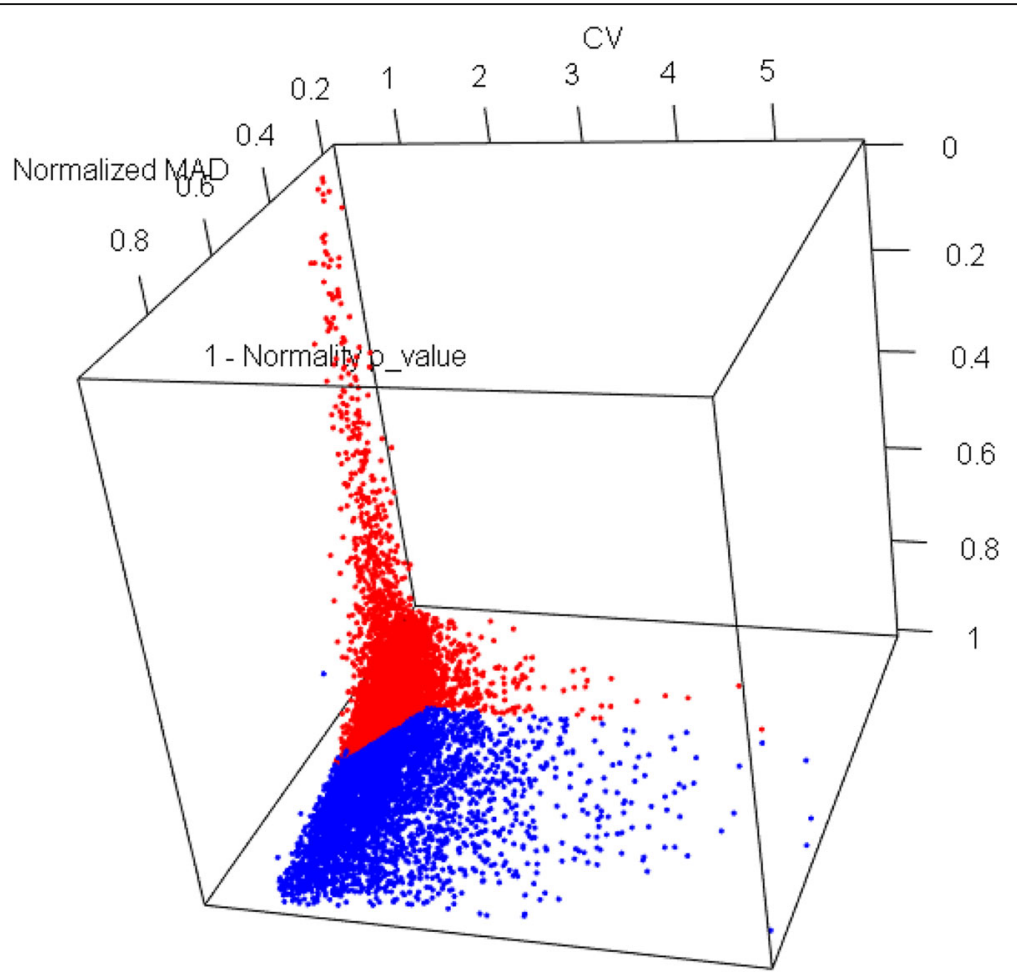

Fig. 1 Cluster of genes in the three-dimensional space of CV, MAD and 1-p obtained using the PAM method. Genes marked in red represent cluster \#1

and $P P 2 A 1$, a protein phosphatase [11], were included in the experimental validation for comparison are mentioned in Table 2.

\section{Selection of primers}

Melting curve analysis of the top ten selected genes using pooled cDNA from various plant parts of cotton as the target, filtered five genes TMN5, TBL6, UTR5B, AT1g65240 and CYP76B6 that met the criteria for good primers. The use of these primers resulted in a single amplification product of expected size with the templates and no amplification (more than $35 \mathrm{Cq}$ ) for non-template controls (Additional file 4). Calculation of primer efficiencies using a five-fold dilution of cDNA for the five reference gene primers gave $r^{2}>0.97$ and efficiency (E) values of 1.9-2.0 (Additional file 2 and Table 3 ). The rest of the primers showed very low primer efficiency.

Table 1 Medoid Z scores of the clusters

\begin{tabular}{lcrll}
\hline Cluster & $\begin{array}{c}\text { Number } \\
\text { of genes }\end{array}$ & \multicolumn{3}{l}{ Z-score values for the medoid in each cluster } \\
\cline { 3 - 5 } & \multicolumn{2}{c}{ CV } & MAD & $1-p$ \\
\hline 1 & 5973 & -0.599 & -0.676 & 0.221 \\
2 & 4061 & 0.426 & 0.933 & 0.223 \\
\hline
\end{tabular}

Expression analysis of candidate reference gene by qPCR Expression of the chosen candidate reference genes were analyzed across all the tissue samples (leaves, square and stem) between transgenic and non-transgenic lines (Fig. 3). Since Lepidoptera does not infect the roots, it was included only for the non-transgenic lines. RNA quality has been shown in Additional file 1. In Fig. 3, TBL6 and UTR5B showed the least variation between the two categories, followed by TMN5 and AT1g65240. The commonly used gene $P P 2 A 1$ although showed a lower median of expression among the study groups, yet showed greater variation between the transgenic and non-transgenic lines. Same trend can be observed with another popular gene $U B Q 14$.

\section{Expression stability of the reference genes in different age category for various plant organs}

We further analyzed the behavior of the reference genes across various plant tissues (leaf, square and stem) and different age categories ( 1 and 3 months old) in transgenic and non-transgenic lines with tissue samples from four biological replicates in each of the organs and age categories. It can be observed from Fig. $4 \mathrm{a}$ and b, that TBL6 and TMN5 showed a uniform expression across various tissues and age categories, suggesting the combination of these genes as optimal when analyzing the expression level of foreign or a transgene. This result is 


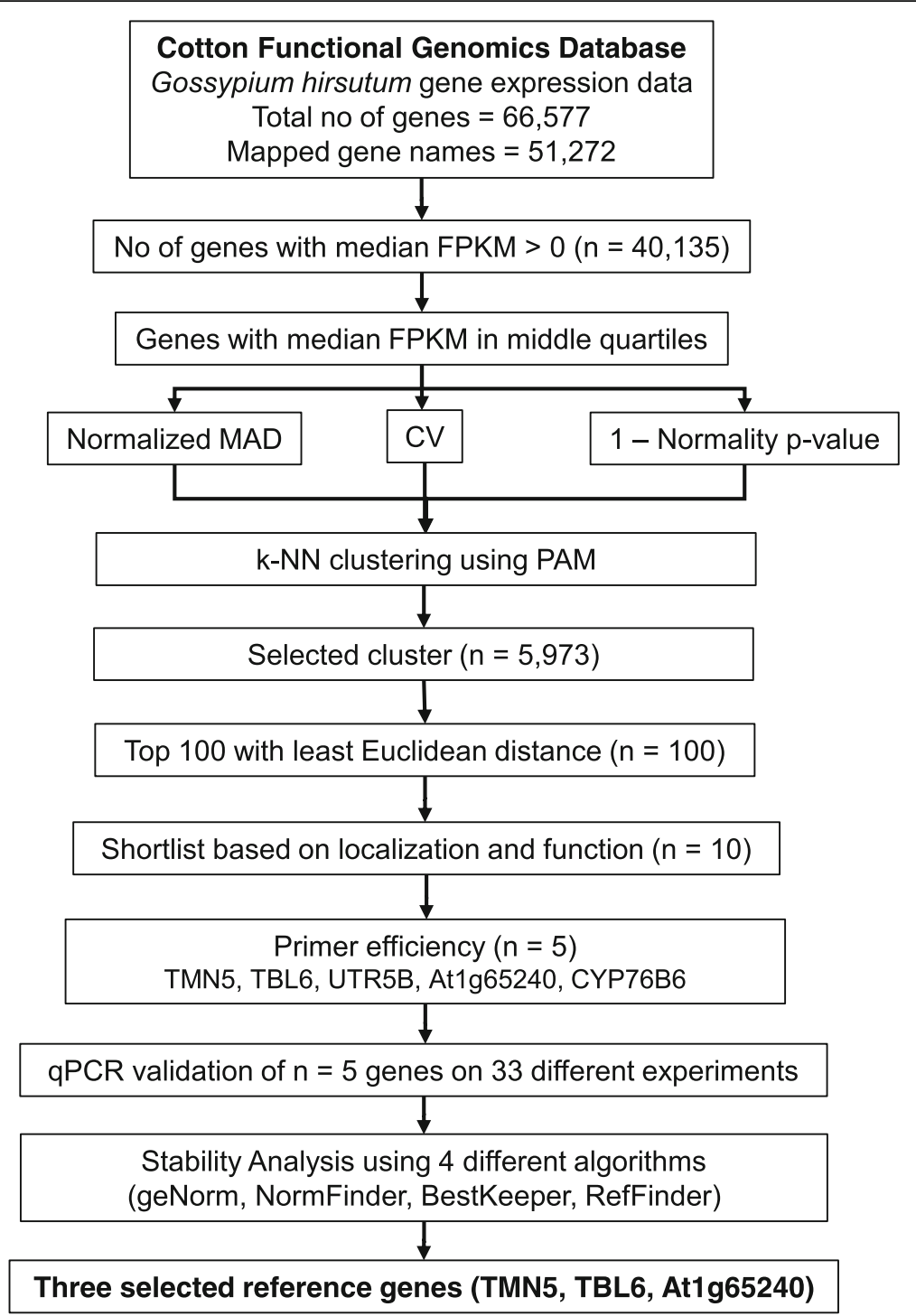

Fig. 2 Work Flow to identify candidate reference genes with least variations and validation of the genes in experiment

also in agreement with the geNorm analysis, which ranked both these genes in the number 1 position, with the least value of stability parameter (Additional file 5).

Expression stability of reference genes across differential developmental stages of leaf and square tissue

Expression analysis of the reference genes during different developmental stages of leaves showed TMN5 (Fig. 5a) to have the least variability in median expression between the mature bottom leaves and young terminal leaves. However, the expression profile of the candidate reference gene in different developmental stages of the square tissues showed a different pattern. CYP76B6 and At1g65240 (Fig. 5b) showed more uniform expression in different developmental stages of the square tissue, whereas TMN5, TBL6 and UTR5B showed greater variability.

\section{Comprehensive stability analysis of candidate reference genes}

Stability of candidate genes was calculated independently by BestKeeper and RefFinder from the raw $C_{p}$ values and by geNorm and NormFinder from relative expression (Additional file 5). The geometric mean of stability ranks assigned in each algorithm was used to create a comprehensive stability ranking of all the candidate reference genes (Fig. 6). The analysis shows TMN5 and TBL6 to be most stable across all tissue types. However, stability analysis including commonly used reference genes from the literature indicates $P P 2 A 1$ at the highest rank followed by TMN5 and TBL6 (Additional file 5). 
Table $\mathbf{2}$ List of selected candidate reference gene for expression analysis and validation

\begin{tabular}{|c|c|c|c|}
\hline Gene.Name & NCBI ref. seq & Description & Function \\
\hline CYP76B6 & $\begin{array}{l}X_{-} \\
016861559\end{array}$ & Geraniol 8-hydroxylase & Heme binding and oxidoreductase activity \\
\hline RPK2 & $\begin{array}{l}X_{-} \\
016855096.1\end{array}$ & $\begin{array}{l}\text { LRR receptor-like serine/threonine-protein } \\
\text { kinase RPK2 }\end{array}$ & Protein Kinase activity \\
\hline At1g65240 & $\begin{array}{l}X_{-} \\
016888563.1\end{array}$ & Aspartic proteinase-like protein 2 & Involved in aspartic-type endopeptidase activity \\
\hline COV1 & $\begin{array}{l}X_{-} \\
016863942.1\end{array}$ & Protein continuous vascular ring 1 & Negatively regulates the differentiation of vascular tissue in the stem. \\
\hline AZG1 & $\begin{array}{l}X M_{-} \\
016863550.1\end{array}$ & Adenine/guanine permease AZG1 & $\begin{array}{l}\text { Transports natural purines and purine analogs. Confers sensitivity to 8- } \\
\text { azaadenine and 8-azaguanine }\end{array}$ \\
\hline EMB8 & $\begin{array}{l}X_{-} M_{-} \\
016834287.1\end{array}$ & Embryogenesis-associated protein EMB8 & Role in embryogenesis? \\
\hline TMK3 & $\begin{array}{l}X M_{-} \\
016813562.1\end{array}$ & Receptor-like kinase TMK3 & Auxin signal transduction, cell expansion, proliferation and regulation \\
\hline UTR5B & $\begin{array}{l}X_{-} \\
016900481.1\end{array}$ & UDP-galactose/UDP-glucose transporter 5B & Sugar transporter \\
\hline TMN5 & $\begin{array}{l}\text { XM_ } \\
016895405.1\end{array}$ & Transmembrane 9 superfamily member 5 & Protein localization \\
\hline TBL6 & $\begin{array}{l}X_{-} \\
016880182.1\end{array}$ & Protein trichome birefringence-like 6 & O-acetyltransferase activity \\
\hline PP2A1 & $\begin{array}{l}\text { XM_ } \\
016840233.1\end{array}$ & Protein Phosphatase 2A & Phosphatase activity \\
\hline UBQ14 & $\begin{array}{l}X M_{-} \\
016867963.1\end{array}$ & Polyubiquitin & Ubiqutination Reaction \\
\hline
\end{tabular}

\section{Discussion}

Reliability of qPCR results are largely dependent on reference genes against which the expression level of the target gene is normalized. Hence, the choice of the reference gene is the most critical parameter while analyzing comparative gene expression studies. Most reliable reference genes should not be regulated across sample types like

Table 3 Primer sequences and efficiency of the shortlisted primers used in this study

\begin{tabular}{llcc}
\hline Gene.Name & Primers (5' to 3') & Efficiency (\%) & $R^{2}$ \\
\hline PP2A1 & F-GATCCTTGTGGAGGAGTGGA & 93.54 & 0.99 \\
& R-GCGAAACAGTTCGACGAGAT & & \\
TMN5 & F-CTCACCATTCCATTACTTGTGTT & 103.24 & 0.97 \\
& R-GAGGAATCTCTCTCGGGTATCT & & \\
UBQ14 & F-CAACGCTCCATCTTGTCCT & 103.74 & 0.99 \\
& R-TGATCGTCTTCCCGTAAGC & & \\
TBL6 & F-AGCAGATCCAGAGACAAGAAAG & 95.14 & 0.99 \\
& R-CCATTGTAGGTGCAGGTGTAT & & \\
UTR5B & F-CGGTCTCTGCTGGTTCTTAG & 94.91 & 0.99 \\
& R-TGACATGTTGTGGTAGGATGT & & \\
At1965240 & F-GCAAACTCTACAGCTCCCATTA & 104.42 & 0.99 \\
& R-GTCCAAACCCGAAGATTCCA & & \\
CYP76B6 & F-TGGCTTGGATGCCTGTT & 103.71 & 0.99 \\
& R-TCGCCGTAAGTGTTGGTTAG & & \\
\hline
\end{tabular}

tissues isolated from different parts of the plant, across different age and developmental stages, various abiotic and biotic stress conditions and different genotypes. However, it is difficult to pinpoint one single reference gene to be optimal for all experimental conditions. As compiled in a

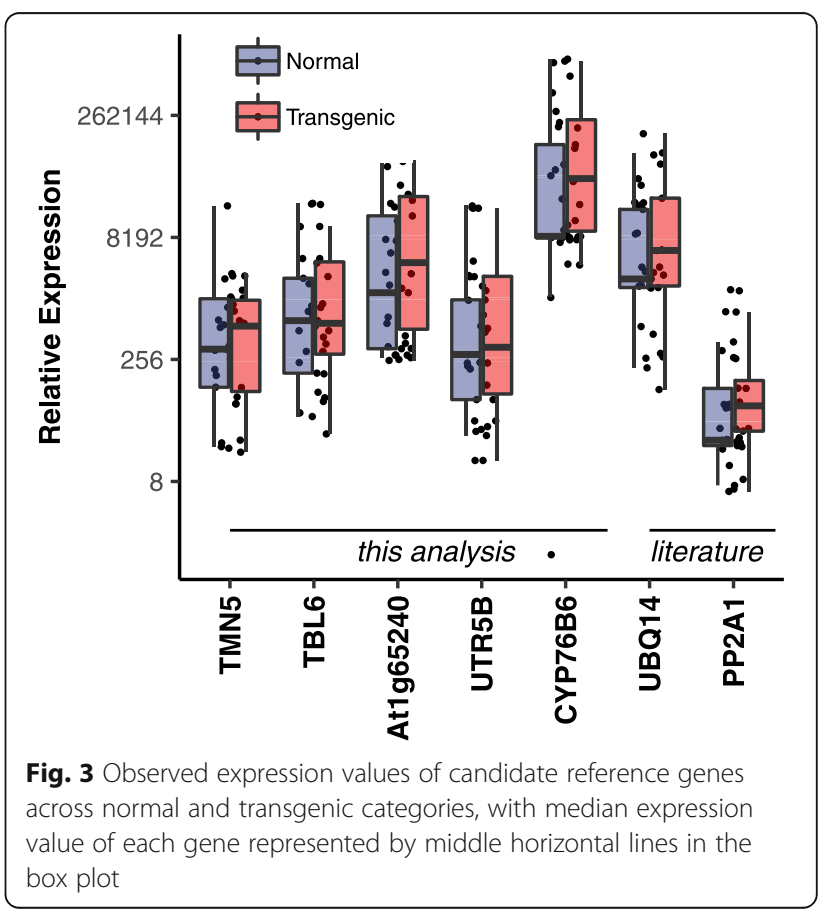



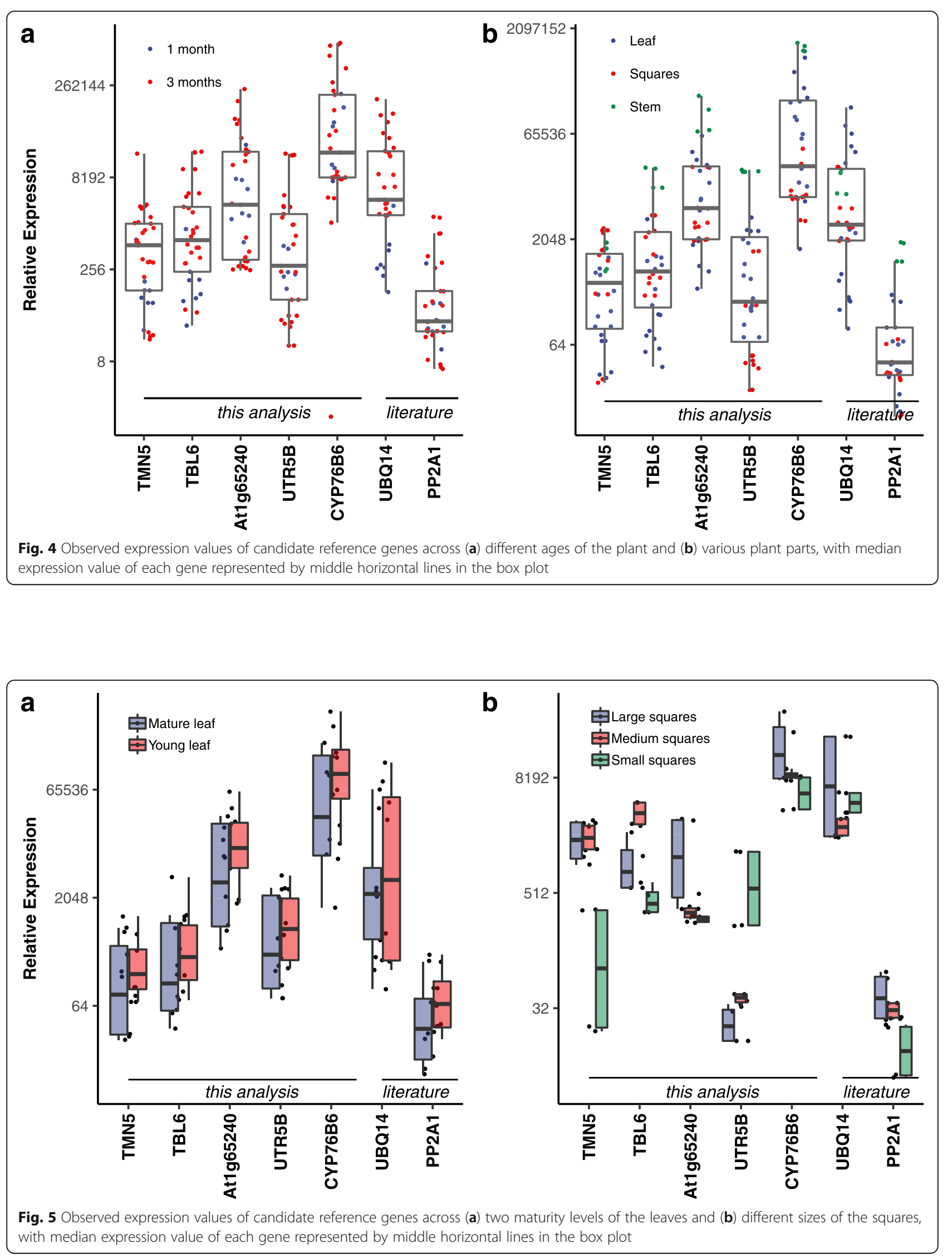


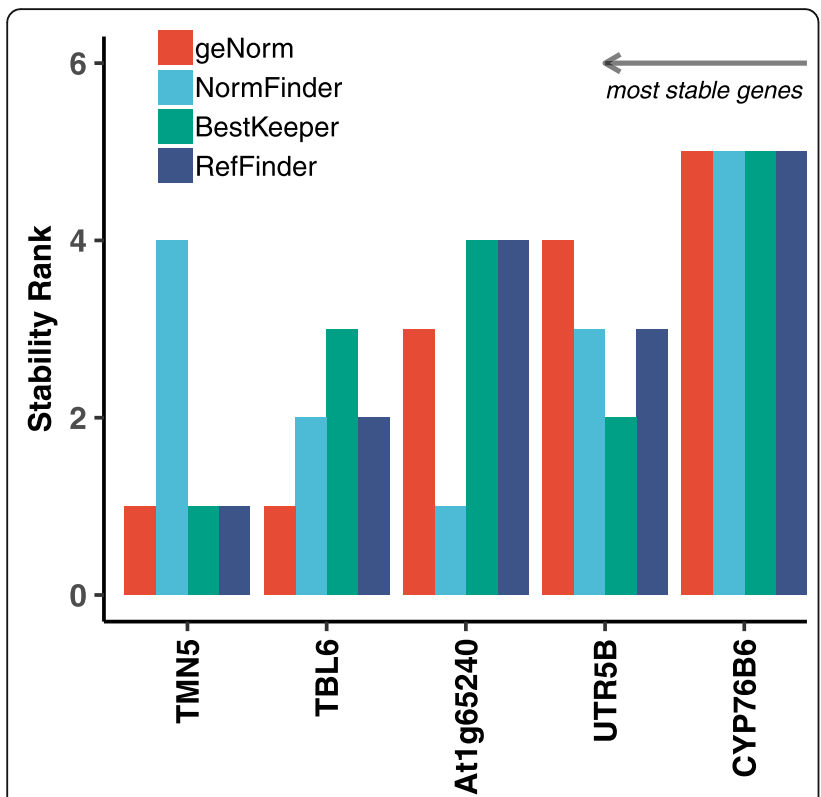

Fig. 6 Stability Ranks of the chosen reference genes candidate using four different algorithms - geNorm, BestKeeper, NormFinder and RefFinder

review paper by Joseph et al. [11] and two recent literatures [24,36], more than 20 reference genes have been used for expression analysis in cotton plants. Fausto et al. [47] have even used miRNA as reference genes for expression of mRNA in cotton. However, all these genes have been chosen based on their function or traditional usage in other systems. The current study is the first attempt of an unbiased genome wide search to find the most stable genes for expression analysis across various tissues and transgenic stress.

Based on the primer efficiency test, we took forward five novel genes for further experiments. Expression stability of these genes was checked by qPCR in experimental sets including different tissues, age categories, developmental stages and different genotypes. Two genes, TMN5 and TBL6 emerged as the most overall stable genes (Fig. 6). Though there is not too much difference between AT1g65240 and UTR5B in overall stability, AT1g65240 showed much lesser variation in squares, one of the target plant organs of Cry gene expression in defence of biotic stress. Thus, in overall stability TMN5, TBL6 and AT1g65240 emerge as clear winners. Among the five genes tested CYP76B6 had the highest expression and least stability, a pattern that is observed in studies with other organisms as well [10].

A probe into the function of these genes revealed that TMN5 encodes for Transmembrane 9 superfamily member 5 protein, which functions in protein localization to the membrane in Arabidopsis thaliana [48]. Interestingly, a recent genome wide search for reference genes in Ciona savignyi, a kind of mollusc, also has identified $T M N$ to be a stable gene [10]. TBL6 encodes for trichome birefringence-like 6 which is involved in acetyltransferase activity in Arabidopsis [49]. The protein coded by AT1g65240 gene, Aspartic proteinase-like protein has been shown to be expressed in seed pods and proposed to play a role in the processing and degradation of the storage proteins in the seeds [50] and thus explaining its high expression levels in the square tissues. Similarly, CYP76B6, geraniol hydroxylase, belongs to the family of CYP76 coding for cytochrome P450 enzyme, which catalyzes the single or double oxidation of all linear mono-terpenols, derivatives of which are particularly found in flower, fruit and young leaves [51]. The protein encoded by UTR5B is involved in carbohydrate transport [52].

Two genes from the literature PP2A1 and $U B Q 14$, when compared on the same experimental platforms with the five genes found in this study, revealed that the lower expressing $P P 2 A 1$ was comparable to TMN5 and TBL6. The protein encoded by PP2A1 is the catalytic subunit of protein phosphatase $2 \mathrm{~A}$ [26], a trimeric holoenzyme, ubiquitously expressed serine/Threonine protein phosphatase, conserved throughout eukaryotes. Though the holoenzyme, among many functions, is involved in regulation of pathogen response [53], the catalytic subunit showed stability between the nontransgenic and transgenic cotton plants.

As documented in this study, most stable genes found by this analysis have very diverse functions. Thus, our traditional idea of selecting reference genes based on function does not hold good any longer.

This is the first time, validation of reference genes to be used in qPCR was done combining analysis of RNAseq data and experimental validation for non-transgenic and transgenic cotton plants. This study becomes important as the transgenic cotton plants account for $95 \%$ of the cotton grown in the total cotton growing areas in India [54] and periodic estimation of transgene expression levels is a critical checkpoint, to ascertain the functionality of these transgenic crops. However, this study only covers the expression stability of the reference genes under normal field conditions and does not include the effect of biotic and abiotic stress conditions on the candidate reference genes.

\section{Conclusion}

The present study has employed a data-driven approach for the identification and experimental validation of reference genes to be used for qPCR studies in transgenic and non transgenic lines of Gossypium hirsutum. Out of the five new candidate reference genes TMN5, TBL6, At1g65240, CYP76B6 and UTR5B analyzed, the first three show stable levels of expression in all the conditions tested 
in our experiments and hence stands out to be potential reference genes in cotton species G. hirsutum.

\section{Methods}

Workflow for this study (Fig. 2) comprised of two broad sections - (i) The statistical analysis of publicly available RNA-seq data to identify candidate reference genes with the least variations in expression and (ii) experimental validation of the selected reference genes.

\section{Gene expression data acquisition}

RNA-seq Fragments per kilobase per million reads (FPKM) values for $G$. hirsutum were downloaded from the Cotton Functional Genomics Database (CottonFGD) [40], which to our knowledge was the only publicly available dataset with RNA-seq expression data for G. hirsutum genes. FPKM values inherently contained two normalization factors - (i) for differences in transcript lengths across genes and (ii) for differences in library sizes across experiments. Since in this analysis our aim was to identify stable genes showing least variations across samples, further normalization was not carried out to remove such variations. Recent published literatures on similar transcriptomic analysis for other organisms [10, 21-23] have also used FPKM or TPM values without further normalization.

CottonFGD contained gene expression data of 66,577 genes across 55 different experimental conditions in different experimental groups such as seed germination (time-series and organ comparison), tissue and organ, ovule development (time-series) and stress experiment (time series for hot, cold, salt and PEG treated samples along with control groups). We downloaded the Joint Genome Institute (JGI) assembly [41] annotation of G. hirsutum from the same database and shortlisted the genes only with mapped gene names and chromosome locations.

In any gene expression experiment, low-expressing genes often create problems for downstream analysis as expression values of such genes are often indistinguishable from sampling noise. To eliminate such genes from the analysis, we used a filtering criterion of median FPKM value $>0$ (or in other words non-zero FPKM value in at least 28 out of 55 samples). Genes those passed the above criteria was selected for the statistical analysis (Fig. 2).

\section{Statistical analysis}

To assess the stability of a gene, two measures were adopted - (i) $\mathrm{CV}=\bar{x} / \sigma_{x}$ where $\bar{x}$ and $\sigma_{x}$ are mean and standard deviation of a variable $x$ respectively and (ii) the normality $p$-value measured by the Shapiro-Wilks Test ( $p$-value $<0.05$ indicates the distribution to be away from Normal) [18]. CV, albeit most frequently used, is affected by outliers and hence fails to be a robust measure. To address this, a third parameter - Median Absolute Deviation ( MAD $=$ median $|x-\hat{x}|$ where $\hat{x}$ is the median of $x$ ) [42] was used after normalization with the median. MAD is a measure of the spread of the distribution and is based on medians, thus less susceptible to deviations by outliers.

An ideal set of reference genes should have low or similar statistical variation across samples (represented by low values of CV and MAD) and should behave as much like a normal distribution (high value of normality $\mathrm{p}$-value or low values of $1-\mathrm{p}$-value). Therefore, a k-medoids clustering of genes was clustered based on the values of the three statistical parameters - CV, MAD (normalized to respective $\mathrm{z}$ scores) and $1-$ p-value using the PAM (Partitioning around Medoids) algorithm originally proposed by Kaufman and Rousseeuw [43]. Medoid based clustering approach was chosen over the more commonly used kmeans method to reduce the effect of outlier genes in cluster determination. The optimal number of clusters required is calculated using the Silhouette graphical method of Rosseeuw [44]. Cluster having the lowest medoid values for each of the three parameters was selected and genes in the cluster were ranked using the Euclidean distance $d=\sqrt{C V^{2}+M A D^{2}+(1-p)^{2}}$ (all parameters replaced by their z-scores) in this threeparameter hyperspace. Top hundred genes with the least values of Euclidean distance were selected and their subcellular location and function were analyzed using Gene Ontology annotation.

\section{Selection of commonly used reference genes from literature}

The most commonly used reference genes were shortlisted from literature keeping in mind their frequencies of usage in the published scientific literature on cotton from 2016 till recent. There were no unique keywords that were used by researchers to report reference genes in expression studies. Hence, a very broad methodology was adopted in which all articles in PubMed were searched for the occurrence of any of the terms "reference gene" or "control gene" or "housekeeping gene" along with co-occurrence of the term Gossypium hirsutum anywhere in the title and abstract. The obtained abstracts were manually curated to find the relevant articles that described studies on reference genes specifically in the context of the cotton plant.

\section{Plant material}

Experiments were performed using 1 and 3 months old transgenic (Bollgard II, Monsanto 15,985, an insect 
resistant line) and non-transgenic lines of Gossypium hirsutum plants grown under normal field conditions, with a temperature range of $25^{\circ} \mathrm{C} \pm 4{ }^{\circ} \mathrm{C}$ and natural photoperiod. During the course of the study, there was no biotic or abiotic stress applied to the plants. The organs used were stem, terminal young leaves, bottom mature leaves and squares in different developmental stages (Small: pin and match head squares, medium: square growth midpoint and large: candle squares). Roots were used only from non-transgenic plant. Replicates were decided as per Artico et al. [26]. Briefly, the material was harvested from three different cotton plants to obtain one pool. The procedure was repeated with three distinct plants in order to obtain a second pool, the biological replicate. A total of 33 experimental categories from transgenic and non-transgenic lines were prepared comprising of leaf, squares, root and stem tissues. For each of the statistical analysis performed in various experimental categories, four replicates of each kind of tissues were analyzed. Total RNA was freshly extracted from all the tissues and stored at $-80^{\circ} \mathrm{C}$.

\section{Total RNA isolation and CDNA synthesis}

Total RNA was extracted from fresh tissues as described previously [45] with slight modifications. Briefly, fresh tissues were homogenized to a fine paste in pre-cooled mortar and pestle using $10 \mathrm{ml}$ of extraction buffer $(400 \mu \mathrm{l}$ of $\beta$-mercaptoethanol and $4 \%$ polyvinyl pyrrolidone) per gram of the plant tissue. An equal volume of water saturated-phenol, chloroform and isoamyl alcohol, at a ratio 25:24:1, was added, mixed thoroughly and centrifuged. The separated aqueous phase was extracted with chloroformisoamyl alcohol, followed by the addition of lithium chloride $(\mathrm{LiCl})$ to a final concentration of $3 \mathrm{M}$. After overnight incubation at $-20^{\circ} \mathrm{C}$, the RNA precipitate was re-suspended in $2 \mathrm{M} \mathrm{LiCl}$, centrifuged and washed with $70 \%$ ice-cold ethanol. Pellet was air-dried and dissolved in $500 \mu \mathrm{l}$ of sterile water. The RNA quality and purity were determined using a NanoDrop $^{\text {mi }}$ 2000/2000c Spectrophotometer (Thermo Scientific) and the integrity of RNA was checked by $1 \%$ agarose gel electrophoresis and ethidium bromide staining (Additional file 1).

cDNAs were synthesized by adding $50 \mu \mathrm{M}$ of Oligo (dT) primer, $10 \mathrm{mM}$ of dNTPs (MBT079, HiMedia), AMV RT (NEB, \#M0277S), 1X First Strand Buffer, RNase inhibitor (Applied Biosystems, N8080119) to $1 \mu \mathrm{g}$ of total RNA. The mixture was incubated at $42^{\circ} \mathrm{C}$ for $1 \mathrm{~h}$ following the manufacturer's instruction. Inactivation of the reverse transcriptase was done by incubating the mixture at $80^{\circ} \mathrm{C}$ for $5 \mathrm{~min}$ and the cDNA solution was stored at $-20^{\circ} \mathrm{C}$.
Real-time quantitative polymerase chain reaction (qPCR)

\section{Primer design}

Primers were designed from the top ten selected genes (Table 2) using the primer Quest tool (https://eu.idtdna. com/pages/tools/oligoanalyzer, 24 May 2019, Integrated DNA Technologies, Inc.) for real-time PCR from Integrated DNA technologies using the criteria that the amplified products range from 90 to $110 \mathrm{bp}$ with a Tm of $62 \pm$ $1{ }^{\circ} \mathrm{C}$. Melt curve analysis of the top 10 selected candidate genes was done and primers showing a single amplicon was chosen for further experiments. The primer sets efficiencies of each primer pair were determined by the standard curve method using serial dilutions of the pooled cDNA, using the formula, Efficiency $(\%)=\left(10^{-1 / \text { slope }}-1\right)$ $\times 100$ (Table 3 and Additional file 2).

qPCR was carried out on Roche LightCycler 480 II instrument using KAPA SyBr green Universal kit (Sigma, \#KK4600) using a 96 well or 384 well optical plates (Roche LightCycler 480 Multiwell Plate 96 or 384, clear, C0687653 384 or C1468659). Reaction mixtures contained $1 \mu \mathrm{l}$ of diluted cDNA in water (1:1), $0.2 \mu \mathrm{M}$ of each primer, $2.5 \mu \mathrm{l}$ of KAPA SyBr Green mix, $1.3 \mu \mathrm{l}$ of water in a total volume of $5 \mu$ l. Reaction mixtures were incubated for $10 \mathrm{~s}$ at $95^{\circ} \mathrm{C}$ followed by 45 amplification cycles of $10 \mathrm{~s}$ at $95^{\circ} \mathrm{C} ; 15 \mathrm{~s}$ at $60^{\circ} \mathrm{C}$ and $15 \mathrm{~s}$ at $72^{\circ} \mathrm{C}$. For each of the sample, the qPCR was performed in technical replicates of three. Three negative controls in which the cDNA was replaced with nuclease free water were also included for each of the primer pair.

\section{Analysis of gene expression stability}

From Quantification cycle $(\mathrm{Cq})$ values of each gene in qPCR experiments, mean of non-template control (NTC) Cq values were subtracted to obtain $\Delta \mathrm{C}_{\mathrm{q}}=\mathrm{C}_{\mathrm{q}}$ (sample) - Mean $\mathrm{C}_{\mathrm{q}}$ (NTC) and relative expression as $\mathrm{E}^{-\Delta \mathrm{Cq}}$ for each replicate, where $\mathrm{E}$ is the primer efficiency of each gene obtained from standard curve. Geometric mean of expression values of replicates are plotted for the chosen reference genes across different samples as depicted in results.

To analyze stability of expression, we used three distinct algorithms geNorm [13], NormFinder [14] and BestKeeper [15] independently and also the web-based RefFinder [19] tool that integrates all these three algorithms plus the DeltaCT method. geNorm algorithm was run using the SLqPCR R package [46], whereas authorsupplied R package or Excel worksheet was used for NormFinder and BestKeeper respectively. Mean Cq values for each gene for all 33 experiments were used as input for BestKeeper and RefFinder, whereas for geNorm and NormFinder the relative expression values were used. Since NormFinder uses a model-based approach to quantify inter- and intra-group variations, the normal 
and transgenic samples are used as two groups for NormFinder analysis.

Results of BestKeeper algorithm, used independently or as part of RefFinder were comparable. However, the rankings differed since BestKeeper uses correlation coefficient with BestKeeper index as ranking parameter, whereas its RefFinder implementation uses standard deviation to rank the genes. Results of geNorm and Normfinder analysis, independently or within ReFinder tool were not compared as they used different inputs. Comprehensive stability rank of each gene was calculated as the geometric mean of stability rank given by each method.

\section{Additional files}

Additional file 1: Gel image of all the RNA samples used in the study. (DOCX $17394 \mathrm{~kb}$ )

Additional file 2: Primer pair efficiency. (PDF $211 \mathrm{~kb}$ )

Additional file 3: List of Genes selected in the study. The list of genes in cluster 2 (sheet 1) and cluster 1(sheet 2) top 100 Genes in the selected cluster 1 , sorted in ascending order based on the calculated Euclidean distance (sheet 3) and the selected 10 (sheet 4) genes are listed. (XLSX $1375 \mathrm{~kb})$

Additional file 4: Melt curves of the final selected primer. (PDF $861 \mathrm{~kb}$ )

Additional file 5: Stability analysis of the candidate reference genes using different algorithms. (XLSX $69 \mathrm{~kb}$ )

\begin{abstract}
Abbreviations
At1g65240: Aspartic proteinase-like protein 2; AZG1: Adenine/guanine permease AZG1; CDNA: Complementary DNA; COV1: Protein continuous vascular ring 1; Cq: Quantification cycle; CV: Coefficient of variation; CYP76B6: Geraniol 8-hydroxylase; DNA: Deoxyribo nucleic acid; EMB8: Embryogenesis-associated protein EMB8; FPKM: Fragments per kilobase of transcript per million mapped reads; GO: Gene Ontology; JGI: Joint Genome Institute; MAD: Median Absolute Deviation; NCBI: National Center for Biotechnology Information; NTC: Non-template control; PAM: Partitioning around Medoids; PEG: Polyethylene glycol; PP2A1: Protein phosphatase 2A; qPCR: Quantitative real-time polymerase chain; RNA: Ribo nucleic acid; RPK2: LRR receptor-like serine/threonine-protein kinase RPK2; RT-PCR: Reverse transcription polymerase chain reaction; TBL6: Protein trichome birefringence-like 6; TMK3: Receptor-like kinase TMK3; TMN5: Transmembrane 9 superfamily member 5; UBQ14: Polyubiquitin; UTR5B: UDP-galactose/UDP-glucose transporter 5B
\end{abstract}

\section{Acknowledgements}

The authors thank Nehanjali Dwivedi, MSMF, Bangalore for critical review of the manuscript, Joy Kuri, Haresh Dagale and Chandramani Singh, Department of Electronic Systems Engineering, IISC, Bangalore for kindly providing computing infrastructure and critical review of the data analysis procedure.

\section{Authors' contributions}

SPK designed and performed the experiments and drafted the manuscript. AKM and CB helped in growing the plants, sample collection and RNA extraction. ASK and VK helped in performing qPCR. SKD conceived the study, performed the statistical and data analysis and drafted the manuscript. CKN guided the study and MD coordinated the study and critically reviewed literature and the manuscript. All authors read and approved the final text.

\section{Funding}

The present study was funded by Beyond Antibody LLP.

\section{Availability of data and materials}

The datasets analyzed during the current study are available from the corresponding author on reasonable request.

All data generated or analyzed during this study are included in this published article [and its Additional files].

\section{Ethics approval and consent to participate}

Not applicable.

\section{Consent for publication}

Not applicable.

\section{Competing interests}

The authors declare that they have no competing interests.

\section{Author details}

'Department of Biotechnology, Research and Development Centre, Bharathiar University, Coimbatore, Tamil Nadu 641 046, India. ${ }^{2}$ Beyond Antibody LLP, S-005 Krishna Greens, Krishna Temple Road, Dodda Bomasandra, Bangalore, Karnataka 560 097, India. ${ }^{3}$ Tumor Immunology Program, DSRG1, MSCTR, MSMF, Mazumdar Shaw Medical Centre, 8th floor, Narayana Health City, Bommasandra, Bangalore, Karnataka 560 099, India. ${ }^{4}$ School of Bio Sciences \& Technology, Vellore Institute of Technology, Vellore, Tamil Nadu 632 014, India. ${ }^{5}$ Division of Plant Physiology and Biotechnology, UPASI Tea Research Foundation, Tea Research Institute, Nirar Dam, Valparai, Coimbatore, Tamil Nadu 642 127, India.

Received: 28 May 2019 Accepted: 26 August 2019

Published online: 14 September 2019

\section{References}

1. Kozera B, Rapacz M. Reference genes in real-time PCR. J Appl Genet. 2013; 54(4):391-406

2. Sun $\mathrm{R}$, He Q, Zhang B, Wang Q. Selection and validation of reliable reference genes in Gossypium raimondii. Biotechnol Lett. 2015;37(7):1483-93.

3. Bustin SA. Real-time, fluorescence-based quantitative PCR: a snapshot of current procedures and preferences. Expert Rev Mol Diagn. 2005;5(4):493-8.

4. Lü J, Yang C, Zhang Y, Pan H. Selection of reference genes for the normalization of RT-qPCR data in gene expression studies in insects: a systematic review. Front Physiol. 2018;9:1560.

5. Zhu X, Li X, Chen W, Chen J, Lu W, Chen L, et al. Evaluation of new reference genes in papaya for accurate transcript normalization under different experimental conditions. PLoS One. 2012;7(8):e44405.

6. Schmittgen TD, Zakrajsek BA. Effect of experimental treatment on housekeeping gene expression: validation by real-time, quantitative RT-PCR. J Biochem Biophys Methods. 2000;46(1-2):69-81.

7. Thellin O, Zorzi W, Lakaye B, De Borman B, Coumans B, Hennen G, et al. Housekeeping genes as internal standards: use and limits. J Biotechnol. 1999;75(2-3):291-5.

8. Bustin SA, Nolan T. Pitfalls of quantitative real-time reverse-transcription polymerase chain reaction. J Biomol Tech. 2004;15(3):155.

9. Huggett J, Dheda K, Bustin S, Zumla A. Real-time RT-PCR normalisation; strategies and considerations. Genes Immun. 2005;6(4):279.

10. Zhan A, Huang X, Li S. Genome-wide identification and evaluation of new reference genes for gene expression analysis under temperature and salinity stresses in Ciona savignyi. Front Genet. 2019;10:71.

11. Joseph JT, Poolakkalody NJ, Shah JM. Plant reference genes for development and stress response studies. J Biosci. 2018;43(1):173-87.

12. Almeida TA, Quispe-Ricalde A, de Oca FM, Foronda P, Hernández MM. A high-throughput open-array qPCR gene panel to identify housekeeping genes suitable for myometrium and leiomyoma expression analysis. Gynecol Oncol. 2014;134(1):138-43.

13. Vandesompele J, De Preter K, Pattyn F, Poppe B, Van Roy N, De Paepe $A$, et al. Accurate normalization of real-time quantitative RT-PCR data by geometric averaging of multiple internal control genes. Genome Biol. 2002;3(7):research0034.

14. Andersen $\mathrm{CL}$, Jensen $\mathrm{J}$, Ørntoft TF. Normalization of real-time quantitative reverse transcription-PCR data: a model-based variance estimation approach to identify genes suited for normalization, applied to bladder and colon cancer data sets. Cancer Res. 2004;64(15):5245-50. 
15. Pfaffl MW, Tichopad A, Prgomet C, Neuvians TP. Determination of stable housekeeping genes, differentially regulated target genes and sample integrity: BestKeeper-Excel-based tool using pair-wise correlations. Biotechnol Lett. 2004;26(6):509-15.

16. Beer L, Mlitz V, Gschwandtner M, Berger T, Narzt MS, Gruber F, et al. Bioinformatics approach for choosing the correct reference genes when studying gene expression in human keratinocytes. Exp Dermatol. 2015; 24(10):742-7.

17. Bahr SM, Borgschulte T, Kayser KJ, Lin N. Using microarray technology to select housekeeping genes in Chinese hamster ovary cells. Biotechnol Bioeng. 2009;104(5):1041-6.

18. Thomas D, Finan C, Newport MJ, Jones S. DNA entropy reveals a significant difference in complexity between housekeeping and tissue specific gene promoters. Comput Biol Chem. 2015;58:19-24.

19. Xie F, Xiao P, Chen D, Xu L, Zhang B. miRDeepFinder: a miRNA analysis tool for deep sequencing of plant small RNAs. Plant Mol Biol. 2012;80(1):75-84.

20. Carmona R, Arroyo M, Jiménez-Quesada MJ, Seoane P, Zafra A, Larrosa R, et al. Automated identification of reference genes based on RNA-seq data. Biomed Eng Online. 2017;16(1):65

21. MacRae T, Sargeant T, Lemieux S, Hébert J, Deneault É, Sauvageau G. RNA-Seq reveals spliceosome and proteasome genes as most consistent transcripts in human cancer cells. PLoS One. 2013;8(9):e72884.

22. Zhou Z, Cong P, Tian Y, Zhu Y. Using RNA-seq data to select reference genes for normalizing gene expression in apple roots. PLoS One. 2017;12(9):e0185288.

23. Li Y, Zhang L, Li R, Zhang M, Li Y, Wang H, et al. Systematic identification and validation of the reference genes from 60 RNA-Seq libraries in the scallop Mizuhopecten yessoensis. BMC Genomics. 2019;20(1):288.

24. Yi C, Hong Y. Estimating the copy number of transgenes in transformed cotton by real-time quantitative PCR. Transgenic cotton. In: Zhang B. (eds) Transgenic Cotton. Methods in Molecular Biology. New York: Humana Press. 2019;1902: 137-157.

25. Hamid R, Tomar RS, Marashi H, Shafaroudi SM, Golakiya BA, Mohsenpour M. Transcriptome profiling and cataloging differential gene expression in floral buds of fertile and sterile lines of cotton (Gossypium hirsutum L.). Gene. 2018;660:80-91.

26. Artico S, Nardeli SM, Brilhante O, Grossi-de-Sa MF, Alves-Ferreira M. Identification and evaluation of new reference genes in Gossypium hirsutum for accurate normalization of real-time quantitative RT-PCR data. BMC Plant Biol. 2010;10(1):49.

27. Wang $M$, Wang $Q$, Zhang B. Evaluation and selection of reliable reference genes for gene expression under abiotic stress in cotton (Gossypium hirsutum L.). Gene. 2013;530(1):44-50.

28. Zhu Y-N, Shi D-Q, Ruan M-B, Zhang L-L, Meng Z-H, Liu J, et al. Transcriptome analysis reveals crosstalk of responsive genes to multiple abiotic stresses in cotton (Gossypium hirsutum L.). PLoS One. 2013;8(11):e80218.

29. Liang C, Meng Z, Meng Z, Malik W, Yan R, Lwin KM, et al. GhABF2, a bZIP transcription factor, confers drought and salinity tolerance in cotton (Gossypium hirsutum L.). Sci Rep. 2016;6:35040.

30. Lin M, Pang C, Fan S, Song M, Wei H, Yu S. Global analysis of the Gossypium hirsutum L. Transcriptome during leaf senescence by RNA-Seq. BMC Plant Biol. 2015;15(1):43.

31. Evans O, Dou L, Guo Y, Pang C, Wei H, Song M, et al. GhNAC18, a novel cotton (Gossypium hirsutum L.) NAC gene, is involved in leaf senescence and diverse stress responses. Afr J Biotechnol. 2016;15(24):1233-45.

32. Dongdong L, Ming Z, Lili H, Xiaobo C, Yang G, Xingqi G, et al. GhMAPKKK49, a novel cotton (Gossypium hirsutum L.) MAPKKK gene, is involved in diverse stress responses. Acta Physiol Plant. 2016;38(1):13.

33. Meng C, Cai C, Zhang T, Guo W. Characterization of six novel NAC genes and their responses to abiotic stresses in Gossypium hirsutum L. Plant Sci. 2009;176(3):352-9.

34. Huang X-Z, Chen J-Y, Xiao H-J, Xiao Y-T, Wu J, Wu J-X, et al. Dynamic transcriptome analysis and volatile profiling of Gossypium hirsutum in response to the cotton bollworm Helicoverpa armigera. Sci Rep. 2015;5:11867.

35. Ding X, Zeng J, Huang L, Li X, Song S, Pei Y. Senescence-induced expression of ZmSUT1 in cotton delays leaf senescence while the seed coat-specific expression increases yield. Plant Cell Rep. 2019;38:1-10.

36. Cao A, Shao D, Cui B, Tong X, Zheng Y, Sun J, et al. Screening the reference genes for quantitative gene expression by RT-qPCR during SE initial dedifferentiation in four Gossypium hirsutum cultivars that have different SE capability. Genes. 2019;10(7):497.
37. Stam M, Mol JN, Kooter JM. The silence of genes in transgenic plants. Ann Bot. 1997;79(1):3-12

38. Antosiewicz DM, Barabasz A, Siemianowski O. Phenotypic and molecular consequences of overexpression of metal-homeostasis genes. Front Plant Sci. 2014;5:80.

39. Laboulaye MA, Duan X, Qiao M, Whitney IE, Sanes JR. Mapping transgene insertion sites reveals complex interactions between mouse transgenes and neighboring endogenous genes. Front Mol Neurosci. 2018;11:385.

40. Zhu T, Liang C, Meng Z, Sun G, Meng Z, Guo S, et al. CottonFGD: an integrated functional genomics database for cotton. BMC Plant Biol. 2017;17(1):101.

41. Paterson AH, Wendel JF, Gundlach $H$, Guo H, Jenkins J, Jin D, et al. Repeated polyploidization of Gossypium genomes and the evolution of spinnable cotton fibres. Nature. 2012;492(7429):423.

42. Pham-Gia T, Hung T. The mean and median absolute deviations. Math Comput Model. 2001;34(7-8):921-36.

43. Kaufman L, Rousseeuw PJ. Finding groups in data: an introduction to cluster analysis: Wiley; 2009. https://doi.org/10.1002/9780470316801

44. Rousseeuw PJ. Silhouettes: a graphical aid to the interpretation and validation of cluster analysis. J Comput Appl Math. 1987;20:53-65.

45. Sajeevan RS, Shivanna MB, Nataraja KN. An efficient protocol for total RNA isolation from healthy and stressed tissues of mulberry (Morus sp.) and other species. Am J Plant Sci. 2014;5(13):2057.

46. Kohl M. SLqPCR: functions for analysis of real-time quantitative PCR data at SIRS-Lab GmbH. Jena: R Package, SIRS-Lab GmbH; 2007.

47. Fausto AKS, da Franca ST, Romanel E, Vaslin MF. microRNAs as reference genes for quantitative PCR in cotton. PLoS One. 2017;12(4):e0174722.

48. UniProt KB. https://www.uniprot.org/uniprot/F4HW17. Accessed 15 Aug 2019.

49. UniProt KB. https://www.uniprot.org/uniprot/Q9LZQ1. Accessed 15 Aug 2019.

50. Chen X, Pfeil JE, Gal S. The three typical aspartic proteinase genes of Arabidopsis thaliana are differentially expressed. Eur J Biochem. 2002; 269(18):4675-84

51. Ilc T, Parage C, Boachon B, Navrot N, Werck-Reichhart D. Monoterpenol oxidative metabolism: role in plant adaptation and potential applications. Front Plant Sci. 2016;7:509.

52. UniProt KB. https://www.uniprot.org/uniprot/A0A1U8PXU0. Accessed 15 Aug 2019.

53. Booker MA, DeLong A. Atypical protein phosphatase $2 \mathrm{~A}$ gene families do not expand via Paleopolyploidization. Plant Physiol. 2017;173(2):1283-300.

54. Priyadarshini SE, Chakraborty TE. Biotechnology: an agent for sustainable socio-economic transformation-volume on 30 years of Department of Biotechnology, Ministry of Science \& Technology, Government of India. Gurgaon: NPG India; 2016.

\section{Publisher's Note}

Springer Nature remains neutral with regard to jurisdictional claims in published maps and institutional affiliations.

\section{Ready to submit your research? Choose BMC and benefit from:}

- fast, convenient online submission

- thorough peer review by experienced researchers in your field

- rapid publication on acceptance

- support for research data, including large and complex data types

- gold Open Access which fosters wider collaboration and increased citations

- maximum visibility for your research: over $100 \mathrm{M}$ website views per year

At BMC, research is always in progress.

Learn more biomedcentral.com/submissions 